\title{
A SURVEY OF RADIO SOURCES AT 3.5-M WAVELENGTH
}

\author{
B. Y. MILLS \\ Radiophysics Laboratory, Commonwealth Scientific and Industrial \\ Research Organization, Sydney, Australia
}

1. INTRODUCTION

Since their discovery in 1946 , the discrete sources of radio-frequency radiation have provided some of the most difficult problems in radio astronomy. For their effective study, one requires a very highly sensitive radio telescope sufficiently precise for accurate position determination and with sufficient resolution to observe separately the detectable radio sources. These requirements led to the development of the Sydney 3.5-m Cross-type radio telescope, which is a pencil-beam instrument with a beamwidth of about 50 minutes of arc and a sensitivity such that under ideal conditions a source of intensity about $1 / 10,000$ that of the strongest known source, Cassiopeia $A$, is just detectable.

A survey of the southern sky with this instrument has now been completed, the results are being analyzed and catalogs of radio sources prepared: about 1700 are now listed. The operation of the instrument and the method of preparing the catalogs has been described in detail $[1,2]$ and will not be discussed further here. This paper is based essentially on a recently completed catalog [3] covering the declination zone +10 to -20 degrees, which contains 1159 sources, and a much smaller catalog of 39 strong radio sources close to the plane of the Milky Way described in another Symposium paper [4]. These catalogs are examined for possible identifications of radio sources with optically observed objects and for determining as far as possible the spatial distribution of the sources.

One of the most fascinating possibilities opened up by such work is its possible contribution to the study of cosmology. It appears, however, that this contribution is still in the "promising" stage since the data are not yet sufficiently conclusive. To obtain additional data the instrument has been modified to allow measurement of the angular sizes of sources in the range 10 seconds to 1 minute of arc; the modification has recently been completed and the initial observations are now being made. This should provide a means of selecting and estimating the distance of very distant "radio galaxies" of cosmological importance.

\section{IDENTIFICATIONS}

A study of possible identifications of the radio sources with visible nebulae is in progress, using the Palomar Sky Atlas. This is a large undertaking that, to be really useful, requires the collection of information about any 
suspected nebula and, in addition, the measurement of the angular size of the associated radio source. Although a number of interesting coincidences in position have turned up already, this work is far from complete and will not be described here: instead we will be mainly concerned with possible identifications between radio sources and the bright objects listed in the Skalnaté Pleso catalog [5].

Objects examined in this catalog include emission nebulae, novae, planetary nebulae, globular clusters, and galaxies. The situation as regards emission nebulae is not different from that described before $[1,6]$. It may be summarized by saying that over the greater part of the sky bright-emission nebulae are detectable radio sources, but in the strongly emitting nonthermal regions near the galactic center they are either visible in absorption or not at all. This is consistent with a thermal origin of the radiation from freefree transitions in the ionized gas. Only one new possible identification has been noted, between the extended source at galactic longitude 259 degrees and latitude -0.5 and the emission nebulae complex Stromlo 38a and 38b [7]. There is also some evidence of absorption at the position of $M 8$, which had not been noted earlier. Of the novae, planetary nebulae, and globular clusters, only one coincidence in position is noted (between the globular cluster NGC 7089 and the radio source 21-012). However, the position agreement is not at all close and in view of the lack of radio emission from other globular clusters it cannot be regarded as significant.

In the case of the galaxies, however, the situation is different: two reasonably certain identifications have been made and, on a statistical basis, it appears probable that there are several "radio galaxies" among a total of 20 coincidences noted in the catalog. These coincidences have been noted where source and galaxy have positions within $1^{\mathrm{m}}$ in right ascension and 20 minutes of arc in declination, which correspond approximately to three times the mean probable errors in each coordinate. There are 315 galaxies listed in the area of the catalog and it is easily shown that the number of coincidences expected is about 9 if galaxies and sources are completely uncorrelated. The number of actual coincidences, 20 , is therefore significantly greater, but not enough to warrant a detailed analysis. We therefore restrict attention to those galaxies within $0 \mathrm{~m} 7$ in right ascension and 13 minutes of arc in declination, that is within two mean probable errors in each coordinate: there are 14 such coincidences and less than 5 expected by chance. The coincidences are listed in Table I together with a statement of the quality of position agreement and the difference between radio and optical magnitudes, $m_{1.9}-m_{p}$, on the assumption that the source may be identified with the galaxy. The radio magnitude is defined by

$$
m_{\lambda}=-53.4-2.5 \log S_{\lambda},
$$

and for comparison with other data, it is converted to the scale of Hanbury Brown and Hazard [8] at $1.9 \mathrm{~m}$ by adding the factor 0.8 magnitude [9]. The photographic magnitude used is the total magnitude as listed by de Vaucouleurs [10]. 
TABLE I

Possible IDENTifications with BRight Galaxies

\begin{tabular}{|c|c|c|c|c|}
\hline \multirow[t]{2}{*}{ Radio Source } & \multicolumn{2}{|c|}{ Galaxy } & \multirow{2}{*}{ Agreement } & \multirow{2}{*}{$m_{1.9}-m_{y}$} \\
\hline & NGC & Type & & \\
\hline $00-07$ & 157 & Sc & very poor & -.13 \\
\hline $01+03$ & 470 & Sbc & fair & -3.5 \\
\hline $01+03$ & 474 & E0 & good & -4.0 \\
\hline $01+04$ & 533 & E3 & very good & -3.6 \\
\hline $01-06$ & 584 & E3-4 & very good & -1.9 \\
\hline $02-014$ & 1068 & $\mathrm{Sb}_{p}$ & very good & -1.0 \\
\hline $03-04$ & 1417 & S: & good & -2.3 \\
\hline $11-118$ & $4038 / 39$ & $\mathrm{Sc}_{p}$ & very good & -0.7 \\
\hline $12+04$ & 4234 & I & good & -3.6 \\
\hline $12+05$ & 4261 & E2-3 & very good & -3.8 \\
\hline $12-118$ & 4782 & So: & very poor & -4.7 \\
\hline $12-118$ & 4783 & So: & poor & -4.7 \\
\hline $14-019$ & 5792 & $\mathrm{~S}$ & very good & -2.5 \\
\hline $21-125$ & 7171 & $\mathrm{SBb}$ & very good & -2.3 \\
\hline
\end{tabular}

The number of good and very good coincidences is the same as the difference between the actual and chance coincidences, suggesting that most may be real. The galaxies NGC 1068 and NGC 4038/39 are almost certainly associated with the coincident radio sources, since the radio emission in each case is only about 2 magnitudes greater than the mean of the "normal" spirals, and in each case there is a peculiarity compatible with increased emission; e.g., NGC 1068 is known to have strong and broad gaseous emission lines in the nucleus and the galaxies NGC 4038 and 4039 appear to be undergoing a mild collision. No abnormalities are listed among the other galaxies, but this means little, for there seems to be no reason why an optically normal galaxy should not emit substantially more than normal at radio frequencies, an established example is NGC 1316. In one case, NGC 7171, for which a Palomar print is available, there is a loose clustering of faint galaxies with several close to the radio position, one or more of which could well be the radio source: angular size measurements would clearly be useful here.

Taking the coincidences at their face value, the suggestion is clear that a substantial proportion of galaxies may have a slightly abnormal radio emission. On the figures quoted, about one galaxy in thirty emits between two and five magnitudes more than a normal galaxy. While not very much weight can be given to this conclusion, it in no way contradicts the observed lack of correspondence between the majority of the sources and the brighter galaxies. It is necessary to increase the statistical reliability of the identifications by extending the catalog to a larger area and, if possible, by increasing the sensitivity, and it is necessary to measure angular sizes.

One might also mention the IAU sources 09S1A $(09-14)$ and 16N0A $(16+010)$ for which Minkowski has suggested possible identifications with faint galaxies. These are discussed by Dewhirst [11]. 
Finally, coincidences are noted in the catalog between the faint sources $08+010,23+01$, and $23+02$ and the Hydra II, Pegasus I, and Pegasus II clusters of galaxies. These are probably significant since the great majority of clusters that have been investigated for radio emission are associated with radio sources. These and other observations of southern clusters will be discussed elsewhere.

\section{STATISTICS}

It was demonstrated some time ago that the statistical properties of the radio sources close to the galactic plane are very different from those in the remainder of the sky [12]. It can now be seen that the difference arises because of the strong sources concentrated close to the ridge line of the galactic emission [4]. The two types of sources referred to previously as Class I (galactic) and Class II (sources distributed uniformly, presumably extragalactic) will therefore be considered separately.

\section{(a) The Class I Sources}

An analysis of the galactic sources is based mainly on a catalog of 39 strong sources visible on a contour map between longitudes $223-0-13$ degrees and latitudes +4 and -6 degrees [4]. The sources with peak temperatures greater than $1500^{\circ} \mathrm{K}$ (flux densities $>7.5 \times 10^{-25}$ watts $\mathrm{m}^{-2}(\mathrm{c} / \mathrm{s})^{-1}$ ) have been selected: they occur in a total area of 0.26 steradians, the density being about 150 sources per steradian. Later we will see that at high latitudes the density of sources above the same minimum intensity is only about 7 sources per steradian, whence it is safe to assume that the great majority of the 39 sources are galactic. In Table II some of the properties of these are tabulated as a function of galactic longitude. The additional sources between longitudes 200 to 170 degrees have been taken from the main catalog [3] within 6 degrees of the galactic plane. The central complex IAU $17 \mathrm{~S} 2 \mathrm{~A}$ has been excluded from this analysis since it is clearly a special case; it has been discussed elsewhere [13].

\section{TABLE II}

Statistical Properties of the Class i sources with Flux DENSITY GREATER THAN $7.5 \times 10^{-25}$ WATTS $M^{-2}(\mathrm{C} / \mathrm{s})^{-1}$

$\begin{array}{ccc}\begin{array}{r}\text { Longitude } \\ \text { (degrees) }\end{array} & \begin{array}{c}\text { Number of } \\ \text { sources }\end{array} & \begin{array}{c}\text { Mean latitude } \\ \text { (degrees) }\end{array} \\ 358-328 & 9 & -1.34 \\ 328-298 & 10 & -1.23 \\ 298-268 & 8 & -1.05 \\ 268-238 & 7 & -0.46 \\ 200-170 & 4 & -2.50\end{array}$

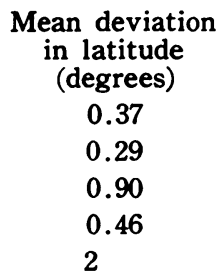

Mean intensity
$10^{-24}$ watts $\mathrm{m}^{-2}(\mathrm{c} / \mathrm{s})^{-1}$
4.4
3.1
3.6
2.9
1.5

It is evident that there is a substantial change in the statistical properties of the sources between the center and anti-center regions. Toward the anti- 
center the sources are less numerous, weaker, and more scattered. Further differences not brought out in the table are:

(1) In the region 200 to 170 degrees all the sources are easily resolvable with the instrument, while between 268 and 358 degrees only 3 of the 27 sources can definitely be resolved, although there are a few others where resolution is suspected; (2) at longitudes less than 260 degrees hydrogen emission nebulae, emitting thermally, are observed in addition to the nonthermal sources; the actual number of nonthermal sources in the interval 268 to 238 degrees is 5 and in the interval 200 to 170 degrees, only 3 .

These observed properties appear consistent with a suggested origin of the nonthermal sources principally in type II supernovae [4]. The sources toward the galactic anti-center, where such supernovae are presumably comparatively rare, might then represent older, weaker, and intrinsically larger objects.

The mean height of the sources above the galactic plane may be estimated from the angular deviations by assigning a mean distance. Taking distances of 8 kiloparsecs in the direction of the center, decreasing to about 2 kiloparsecs in the 200 degrees to 170 degrees region (the mean of the local and suggested next outer arm) the angular deviations indicate a mean height above the plane of, very roughly, 50 parsecs, consistent with a Population I distribution. However, no such data relating to the type II supernovae is available for comparison. Further discussion of these Class I sources and their possible relation to the other galactic components is given in another paper [4].

\section{(b) The Class II Sources}

Two properties of the distribution of Class II radio sources in the main catalog area have been investigated statistically: these are the two-dimensional distribution across the celestial sphere and the distribution in depth based on counts to different flux-density levels. Because of the finite resolution of the aerial, spatial clustering may affect the source counts substantially; clustering is therefore dealt with first, principally by considering the two-dimensional distribution and some observations of source sizes and background irregularities.

It was suggested earlier that the radio sources might display large-scale clustering [1]. This conclusion was based essentially on applying the $x^{2}$ test to areas measuring $10 \times 10$ degrees; the distribution was found to be nonrandom at the 2 per cent confidence level. We now have a much greater quantity of more homogeneous data, and similar tests that have been applied indicate that large-scale deviations from randomness are much less than before and perhaps are not significant. The $\chi^{2}$ test was applied to sources above various limiting intensity levels in areas of different sizes well away from the galactic circle. It is only with $30 \times 30$-degree squares that a significant nonrandomness is indicated. This applies to all sources, and sources stronger than $10^{-25}$ watts $\mathrm{m}^{-2}(\mathrm{c} / \mathrm{s})^{-1}$; in both cases, however, the confidence level is only 5 per cent. It appears that large-scale clustering. 
if present, is small; with the accumulation of more data it may be useful to apply more sophisticated tests.

It was also noted that the number of "extended" sources listed in the catalog was significantly more than expected from chance blending effects in a universe of randomly distributed "point" sources [1]. This conclusion is strengthened by analysis of the present catalog.

We have considered areas from $21^{\mathrm{h}}-00^{\mathrm{h}}-05^{\mathrm{h}}$ and from $09^{\mathrm{h}}-15^{\mathrm{h}}$, which are well away from the galactic circle. Of the sources with flux densities greater than $40 \times 10^{-26}$ watts $\mathrm{m}^{-2}(\mathrm{c} / \mathrm{s})^{-1}, 20$ are listed as "extended" and 2 as "perhaps extended"; of sources stronger than $20 \times 10^{-26}$ watts $\mathrm{m}^{-2}(\mathrm{c} / \mathrm{s})^{-1}$, 36 are listed as "extended" and 17 as "perhaps extended." The numbers of chance blends classified as a single source that is "extended" or "perhaps extended" may be estimated as before. The expected number of such blends that have flux densities greater than $40 \times 10^{-26}$ watts $\mathrm{m}^{-2}(\mathrm{c} / \mathrm{s})^{-1}$ is 2 , and the number greater than $20 \times 10^{-26}$ watts $\mathrm{m}^{-2}(\mathrm{c} / \mathrm{s})^{-1}$ is 15 . These numbers are very much smaller than the ones observed. It is therefore concluded that a substantial proportion of the stronger sources have an angular size that is resolvable with our aerial $(>\$$ degree) and/or that small scale clustering effects are significant.

Both these explanations are consistent with present astronomical knowledge. It is now commonly accepted, for instance, that the majority of galaxies are organized into clusters of various sizes, and in a large cluster the conditions would seem favorable for the production of radio sources by collision: thus, the existence very close together of several physically related radio sources is quite conceivable. There is also evidence for clustering of a higher order into "supergalaxies" (for example de Vaucouleurs [14]), to which the same arguments apply. Clusters of galaxies in general emit much more at radio frequencies than the integrated emission of their component normal galaxies (e.g., Hanbury Brown and Hazard [15]). This may be attributable to associated "radio galaxies," or sometimes equally well to radiation from the cluster as a whole, that is, to intergalactic emission. The latter process has been discussed in some detail by Shklovskii [16], in its application to radiation from the "local supergalaxy." Thus, on present evidence, it would seem quite possible that individual radio galaxies should appear relatively frequently in physically related groups of two or more, and that close clusterings of galaxies can create vast radio sources of large angular size even at very great distances.

A further possibility that should not be ignored is that some, at least, of the Class II sources of large angular size may be located in our Galaxy. Analysis of variations in brightness of the background radiation observed with the $3.5-\mathrm{m}$ cross aerial shows that some bright regions at moderate distances from the plane are probably related to the galactic emission [4]: obvious examples in the present catalog (not, of course, included in the area analyzed) are the large sources associated with the nebulosities in Orion. At the moment it is sufficient to note that a model comprising a random distribution of "point" sources is inadequate to explain all the observational data. 
In order to investigate the distribution of the radio sources in depth, source counts have been made to various limiting flux-density levels. The counts are shown in Fig. 1, where the logarithms of the number density of sources with flux densities $S$ or higher are plotted against $\log S$ : the standard errors in the plotted points due to chance effects in the distribution $(\sqrt{N})$ are shown as vertical wings in the figure. The actual numbers from which the diagrams were constructed are given in Table III.

TABLE III

The Numbers of Class II Sources Above Defined Flux Density Levels

$$
\begin{gathered}
\text { Flux Density } \\
\left(10^{-26} \text { watts } \mathrm{m}^{-2}(\mathrm{c} / \mathrm{s})^{-1}\right)
\end{gathered}
$$

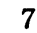

10

20

40

80

160

$\begin{array}{cr}\text { Numbers of Sources } \\ N \\ \text { All sources, } & 1003 \\ & 982 \\ 754 \\ 218 \\ 63 \\ 19 \\ 4\end{array}$

The straight line shown in Fig. 1 passing through the derived points has a slope of -1.8. As discussed earlier [1], there are two instrumental factors that contribute to this slope, the finite resolution of the aerial and the un-

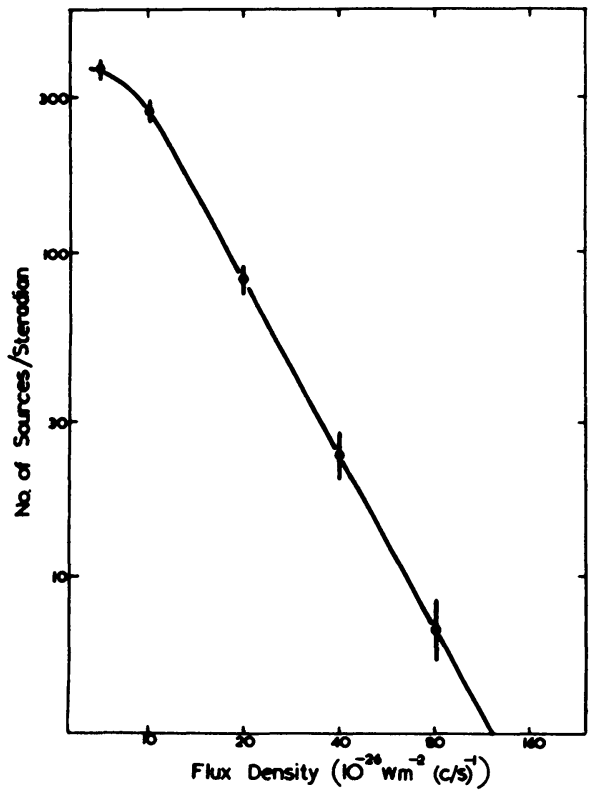

FIG. 1. Counts of Class II sources. certainties due to noise in the weaker flux densities. However, with randomly distributed sources neither effect is large; from the earlier data, a mean increase of slope of about -0.15 is estimated, leaving a net slope of -1.65 . It is well known that, after all corrections, the slope should be -1.5 for a random distribution of "point" sources in a static Euclidean universe; thus there is some additional evidence from the source counts that the above model is inadequate. However, the evidence is not conclusive, for inspection of Fig. 1 shows that the standard errors due to statistical effects are large and the apparent small excess of faint sources could equally well be a statistical deficiency of close and strong sources.

It is interesting to compare the evidence from the angular-size data and 
the source counts to see (1) if an excess of faint and distant sources, corresponding to a slope of -1.65 in the source counts ogive, can increase the number of blends sufficiently to account for the excessive number of "extended" sources, or (2) if a large number of "extended" sources can result in an increased apparent slope of the magnitude observed. It is easy to see from the earlier data that (1) is not possible; the increase in the number of blends would be negligible, since the parameters of the model source distributions were fixed largely by the numbers of faint sources. On the other hand it is evident that if the clustering is greater than in a random distribution the increased slope due to blending will be enhanced; quantitatively the attribution of all extended sources to blends is adequate to explain the observed slope. It might appear that sources of finite angular size could have no effect on the slope, since integrated fluxes are used in the counts. However, the possibility arises because the catalog is restricted to sources of angular size less than 2 degrees, in order to eliminate background irregularities as much as possible; it seems that, at the same time, many large-scale extragalactic concentrations are excluded.

In principle, we may investigate the importance of these factors by comparing the proportion of extended sources listed at different flux-density levels. The bottom end of the catalog must be excluded from comparisons of this kind because of the difficulty of identifying an extended source: the lowest level at which consistent recognition seems possible is $40 \times 10^{-26}$ watts $\mathrm{m}^{-2}$ $(\mathrm{c} / \mathrm{s})^{-1}$. We have seen that the number of extended sources above this level is 20, with 2 listed as "perhaps extended," in a defined area well away from the galactic circle. The total number of sources above the same level in the same area is 46 ; whence it would appear that about half the sources are either of large angular size or physical blends. For sources with flux densities greater than $160 \times 10^{-26}$ watts $\mathrm{m}^{-2}(\mathrm{c} / \mathrm{s})^{-1}$, which, on the average, are at half the distance, the number of extended Class II sources listed in the whole southern sky is 3 out of a total of 10 sources (excluding the Magellanic Clouds as belonging to the class of "normal" galaxies with which we are at present not concerned). Unfortunately the numbers are too low to permit a firm conclusion although they are consistent with some systematic omission of very large sources. The data are obviously inadequate to correct for such effects in order to derive counts dependent only on the large-scale distribution in depth, which is essential before they may usefully be applied to a cosmological model.

Since the principal uncertainty is in the proportion of distant sources of small angular size, the most obvious first step is to examine all the sources with an interferometer having sufficient spacing between aerials to respond to the distant sources alone. Such an instrument, in which the aerial spacing is $3000 \lambda$ yielding a lobe separation of 1.2 minutes of arc, has just recently been put into operation at the Radiophysics Laboratory; the sensitivity appears comparable with that of the survey. A number of very small sources have been observed, but is too early yet to quote any statistics. 
[1] Mills, B. Y., and Slee, O. B. Aust. J. Phys. 10, 162, 1957.

[2] Mills, B. Y., Little, A. G., Sheridan, K. V., and Slee, O. B. Proc. I.R.E. 46, 67, 1958.

[3] Mills, B. Y., Slee, O. B., and Hill E. R. Aust. J. Phys. (In press.)

[4] Mills, B. Y. Paper 79.

[5] Bečvář, A. Atlas Coeli Skalnaté Pleso. Prague (Přirodovědeckě Vydavatelství), 1951.

[6] Mills, B. Y., Little, A. G., and Sheridan, K. V. Aust. J. Phys. 9, 218, 1956.

[ 7] Gum, C. Mem. R.A.S. 68, 155, 1955.

[8] Brown, R. H., and Hazard, C. Phil. Mag. 43, 137, 1952.

[9] Mills, B. Y. "Radio frequency radiation from external galaxies," Handbuch der Physik, Vol. 53. (In press.)

[10] de Vaucouleurs, G. "A revision of the Harvard survey of bright galaxies," Australian National University Mimeograph, Canberra, 1953.

[11] Dewhirst, D. W. Paper 92.

[12] Mills, B. Y. Aust. J. Sci. Res. A 5, 266, 1952.

[13] Mills, B. Y. The Observatory, 76, 65, 1956.

[14] de Vaucouleurs, G. Vistas in Astronomy, Vol. II, Section 15. London and New York (Pergamon Press), 1955.

[15] Brown, R. H., and Hazard, C. M.N.R.A.S. 113, 123, 1953.

[16] Shklovskii, I. S. A. Zh. 31, 533, 1954.

\section{Discussion}

Ryle: I should like to raise the question of the large fraction of extended sources which Mills has reported in the range $S>40 \times 10^{-26}$. In our observations, both at 81.5 and at $159 \mathrm{Mc} / \mathrm{s}$, we made a detailed search for sources of this order of flux having diameters in the range up to one or two degrees, and found a very much smaller proportion than that reported by Mills. I should like to ask him whether he has included sources of greater angular size, which we have excluded as part of the galactic structure. We have excluded any structure so faint as to be below the limit of the survey if so far removed that it could be contained within the interference pattern of the high-resolution system.

Mills: We define a radio source in such a way that the same class of object is counted whether the source is strong and close or weak and distant. If anything, we miss some large and close objects because we list only those less than 2 degrees.

Ryle: We do not base our results simply on the deflections observed in the system of high resolving power; we also have available the observations taken with the interferometer of intermediate resolving power and the total-power observations made with a single element. The purpose of these observations has been to search for sources that might affect the numberintensity counts and the corresponding statistical method of analysis.

Hazard: The combined total-power and interferometer survey made at 90 $\mathrm{Mc} / \mathrm{s}$ at Jodrell Bank shows that there is a considerable number of largeangular diameter sources at high galactic latitudes, confirming the observations made by Mills. The majority of these sources are fairly intense but do not appear in the Cambridge $2 \mathrm{C}$ survey. 